\title{
LA NOVELA ESPAÑOLA EN LA ITALIA DEL SIGLO XVII: EL CASO DE IL FENISO DE FRANCISCO DE QUINTANA ${ }^{1}$
}

\author{
Andrea Bresadola \\ Università degli Studi di Udine \\ andrea.bresadola@uniud.it
}

Será razón que tengas alegría grande, gloria apacible y increíble regocijo

(Quintana, Experiencias de amor y fortuna)

Florencia, estadio Artemio Franchi, Curva Fiesole, 20/10/2013

$\mathrm{L}$ a primera edición de la novela Experiencias de amor y fortuna de Francisco de Quintana se imprimió en 1626 (aunque fue escrita en 1624) y salió a nombre de Francisco de las Cuevas, seudónimo bajo el cual se ocultaba el autor (Quintana 1626). Su traducción italiana se publicó en cambio en 1654, con el título: Il Feniso, overo Avvenimenti d'Amore e di Fortuna portato dalla lingua spagnuola da Bartolomeo Dalla Bella (Quintana 1654). No conocemos mucho sobre esta obra: según mis datos, no existen estudios que excedan la dimensión de las entradas de catálogo ${ }^{2}$.

El estudio de las traducciones puede arrojar luz sobre la presencia de la literatura española en la Italia del siglo XVII: en efecto, la existencia y la difusión de libros como el de Dalla Bella contribuyen a medir el grado de conocimiento de las letras españolas fuera de sus fronteras, puesto que en Italia el circuito de lectores de obras en español era muy limitado (Mazzocchi 2005: 393-394). Además, la técnica de traducir - es decir, verter a otra lengua y a otra tradición un texto en una operación que nunca es neutra — nos brinda informaciones sobre el modo de trueque y adaptación al gusto de destinatarios distintos. Refiriéndose precisamente a

1 Este trabajo se inscribe en el marco del Proyecto I+D+i del MINECO Novela corta del siglo XVII: estudio y edición (y II) (FFI2013-41264-P).

2 La citan ya Antonio (1996, I: 418 col. a; 463, col. b) y Mazzucchelli (1760, II, parte II: 632), si bien este con el título equivocado de Il Cheniso o vero avvenimenti d'amore e di fortuna, que Albertazzi (1891: 208) transformó en Il Chemiso. Aparece también en el fundamental repertorio de Mancini (1970: 265). 
las traducciones narrativas del siglo XVII, Getrevi (1986: 309) definía al traductor italiano, en general, como un «mediatore in un duplice senso: all'interno dell'itinerario del testo e all'interno della dinamica editoriale e culturale».

\section{EXPERIENCIAS DE AMOR Y FORTUNA}

Francisco de Quintana (?-1658) fue un predicador, poeta y prosista que desarrolló su obra durante el primer tercio del siglo XVII (Zimič 1975: 169-189; Quintana 2011: 8-13). En su juventud tuvo que ser bastante conocido en el ambiente poético de Madrid, donde frecuentó a escritores como Pérez de Montalbán, Tirso de Molina, Calderón de la Barca, María de Zayas y Juan de Piña. Fue también íntimo de Lope de Vega en los últimos años de vida del Fénix, asistiéndole en sus postreras horas (Bresadola 2012). Su producción narrativa se limitó a un trienio más que circunscrito, que va aproximadamente de 1624 a 1627. Durante esos años compuso dos novelas, muy parecidas en estilo, contenido y estructura: además de Experiencias de amor y fortuna, editó en 1627 la Historia de Hipólito y Aminta ${ }^{3}$.

La novela de la que nos ocupamos disfrutó de diez ediciones en el siglo XVII y de otra en el XVIII (Quintana 2011: 17-19). Representa bien ese momento de evolución y ebullición del género, cuando la estructura y los temas de la llamada novela «heliodoriana» se funden con los hábitos de la narrativa cortesana y del teatro urbano. Ambientada en un momento muy cercano a la época de su escritura, cuenta la historia del joven noble Feniso y sus andanzas por España, Italia y Argel. En su peregrinatio se topa con varios personajes que le narran su vida, centrándose en el episodio (de amor, honor o fortuna) clave de su existencia. La novela se compone así de distintas «experiencias», apuntadas ya en el título, que desmenuzan la trama principal en una cadena de cuentos de gran extensión, casi como si fuera una colección de novelas cortas.

A pesar de esta variedad, asistimos a una constante elevación estilística: las figuras retóricas y los preciosismos sintácticos contaminan toda la narración y, a veces, manifiestan por parte del autor el lucimiento de sus destrezas. En su alarde retórico, Quintana aprovecha todo el bagaje expresivo de la literatura áurea, revelando especialmente la deuda con la moda gongorina y la oratoria (Quintana 2011: 82-92).

La novela alcanzó cierta resonancia: se hicieron traducciones al francés, al inglés y, finalmente, al italiano. No es casual que la versión francesa (Quintana 1636) fuera la primera, prueba evidente de una significativa atención de esa cultura hacia la literatura española. El hecho es particularmente llamativo porque se produce en un momento en que los dos estados sostenían una guerra. Esto confirma también la mayor difusión de la novela larga en Francia respecto al resto de países del

Este último ha sido objeto de la tesis doctoral de Lepe García (2013a), quien ha publicado ya un resultado (Lepe García 2013b) de sus notables investigaciones. 
continente. En 1651 vería la luz la traducción inglesa (Quintana 1651), que no se hizo a partir del original, sino del francés (Sánchez Escribano 1987: 195).

\section{Il Feniso overo Avvenimenti d’Amore e di Fortuna}

Como vemos, la traducción italiana es la última en orden temporal y apareció después de su difusión en ámbito europeo. Apenas disponemos de noticias sobre el traductor Bartolomeo Dalla Bella, ya que ignoramos también sus fechas de nacimiento y muerte. Los repertorios consultados se limitan a indicar que perteneció a la orden dominica. Dolcetti (1978, I: 38) informa de que su familia era de origen florentino, aunque parece que nació en Venecia, ya que Cornelio (1749: 262) lo define como «venetus». En 1652 figura como prior del convento veneciano de los Santissimi Giovanni e Paolo, donde residía al menos desde 1633, según leemos en la portada de otra traducción suya (Avendaño 1633). Además de la novela de Quintana, en efecto tradujo del español tres obras devotas, todas impresas en Venecia: dos de Cristóbal de Avendaño, Materiale Perfettissimo delle Feste Ordinarie (1633 y 1636) y Prediche nella solennità di Christo (Avendaño 1649); y una de Diego Niseno, La sete che pati Cristo (1634). No contamos con elementos para establecer cómo y dónde Dalla Bella llegó a aprender el castellano, el idioma extranjero, por cierto, más difundido en la Italia del Seiscientos.

La traducción de una obra de narrativa se antoja, entonces, solo una actividad ocasional para él. Esto era muy común en aquel panorama italiano: se registran pocos traductores en los catálogos con más de un título de novelas; y aún menos exhiben un interés duradero hacia una forma literaria determinada. Como ya subrayó Mancini (1970: 206), los traductores italianos de las novelas del Barroco no siempre son conocidos, sino que son «talvolta sconosciuti, talaltra di nessuna o quasi nessuna risonanza». Además, Dalla Bella no era un escritor, no perteneció a ninguna academia veneciana y no compuso o tradujo, según hemos visto, otras obras literarias stricto sensu $\mathrm{s}^{4}$.

El mismo hecho esporádico se halla en relación con las figuras de los tipógrafos de la traducción: Combi-La Noù, es decir, el veneciano Sebastiano Combi (el joven) y el holandés Giovanni de la Noue. Los dos se asociaron en los años cuarenta y hacia la mitad de siglo llegaron a convertirse en una de las mayores organizaciones del comercio librero en el continente (Mirto 1989). No sorprende que la novela se imprimiese en Venecia, quizá el mayor centro de traducción y difusión de la novela barroca española, a pesar de la crisis del mercado editorial del siglo XVII (Cinti 1984: 577-581; Santoro 2007: 445-449). La especial inclinación hacia el género y, más en general, hacia la literatura barroca española en la sociedad de la Serenissima, favoreció la composición de traducciones, adaptaciones,

4 Jöcher (1960, I: col. 928) lo define como «italianischer poet», pero la afirmación parece una errata. 
imitaciones y reelaboraciones por parte de los novelistas venecianos (Vuelta García 2011: 277-281). Sin embargo, Combi-La Noù se mostraron poco aficionados al género. Su catálogo (Griffante 2006, II: 424-425) revela un neto predominio de obras religiosas, a las que siguen, por cantidad, las eruditas, técnico-científicas e históricas. Publicaron muy pocas novelas: Giuochi di fortuna de Assarino, La Vergine parigina y la Heroina intrepida de Frugoni y algunas traducciones, sobre todo del francés (La Giulia de Camus, el Mitridate de Vayer de Boutigny y la Arcadia de Philip Sidney, que no se tradujo del inglés sino del francés, como informa la portada). Estos datos atestiguan una escasa planificación, y afirman también que su atracción hacia la narrativa era independiente de la lengua y de la procedencia de la novela y respondía únicamente a razones comerciales. Todo el repertorio (con la posible excepción de la escandalosa Vergine de Frugoni) no se aleja de una tendencia común a los grandes editores venecianos de la segunda mitad del siglo, poco dispuestos a arriesgarse con libros heterodoxos o con proyectos experimentales de incierta venta (Ulvioni 1977: 108-111).

No son muchas las traducciones del español que salieron de sus tórculos. Las pocas que imprimieron se encargaron siempre a traductores diferentes y siguen la corriente más difundida en Italia, o sea, las obras religiosas (fray Luis de Granada, Santa Teresa de Jesús, José de Jesús María, Alfonso Rodríguez, Francisco de Sigüenza). La novela de Quintana, pues, es un unicum. Il Feniso, además, tuvo una sola edición, y es posible que no circulara demasiado, si pensamos que a día de hoy se conoce solo un ejemplar en todas las bibliotecas públicas italianas: el de la Comunale Manfrediana de Faenza (signatura 10222$)^{5}$.

El punto de partida ideal para dilucidar la relación entre el original y la traducción es el paratexto, que representa el primer puente entre la cultura de partida y la de llegada. Aquí también — como ocurre a menudo (Mazzocchi 2005)— se configura como espacio de diferencia y contraposición, indicio de una falta de homologación entre la realidad literaria española y la italiana. En primer lugar, desaparece del todo el nombre de Quintana, tanto en la portada como en los preliminares. En ningún momento se avisa de quién es el autor de la novela, según una práctica bastante común, sobre todo cuando el autor no gozaba de fama fuera de su patria. Por la misma razón, probablemente, faltan también los elementos paratextuales firmados por Dalla Bella, que no tenía renombre ni como traductor ni como novelista, y por eso no podía contribuir a la venta del libro ${ }^{6}$.

Michel (1967-1984, V: 21) ofrece esta descripción: «-12, [4ff.,] 496 p., frontisp.». El catálogo de la Manfrediana registra que la portadilla calcográfica fue dibujada por Francesco Ruschi y grabada por Giacomo Pecini. He localizado otro ejemplar en la Biblioteca de la Real Academia Española de Madrid (signatura: C-3093) y uno más en la Bibliothèque de l’Arsenal de Paris (FRBNF39336819).

$6 \quad$ En los preliminares de las Prediche nelle solennità di Christo, en cambio, Dalla Bella toma la palabra en «Il tradottore al lettore». Aquí expresa las convencionales limitaciones de la 
Al rico paratexto español se contraponen, escuetos, los del Feniso. En la obra española leemos, por lo menos en la princeps, un carteo entre Lope y el autor, varias anotaciones (entre las cuales una de Tirso de Molina), tres poemas (de Juan Pérez Montalbán, Frutos de León y Tapia y María de Zayas) y un aviso de Quintana al lector. En la versión italiana no queda nada de todo eso: solo figura una dedicatoria convencional de Giacomo De Rossi —o Rossi, que fue durante años agente de Combi (Barbierato 2005: 151) — a Sebastiano Patavino, fechada el 15 de febrero de 1654, y un brevísimo párrafo de «lo stampatore a chi legge».

El cambio es evidente ya en la tipología de los prologuistas: no son célebres escritores los que prestan su pluma para elogiar la novela, sino los mismos responsables del mecanismo de producción y difusión del libro. Por tanto, en el paratexto italiano se desplaza el foco a aspectos de orden casi exclusivamente comercial. No queda rastro de los argumentos que llenan las páginas del original, como la disquisición teórica acerca de la urgencia moral, la ejemplaridad de la obra, la búsqueda del justo medio y la celebración de la elocuencia del autor. Se reducen también a una simple oración en la dedicatoria las acostumbradas aseveraciones sobre la verosimilitud: «chiunque lo scorse in altra lingua attesta esser stato lungamente dubioso se fosse racconto di veri successi o no di menzogne colorite da romanzatrice penna» ${ }^{7}$.

Las diferencias en los paratextos ofrecen también alusiones implícitas sobre el posible destinatario. En los preliminares de Experiencias de amor y fortuna se presentaba la obra como producto «culto» y se prefiguraba un lector «estudioso y entendido», el «docto» y «cuerdo», capaz de captar las numerosas sentencias y las finezas filosóficas dispersadas a lo largo del texto. Por eso en la carta al lector de Quintana abundan las citas de auctoritates clásicas y se insiste en la presencia de las digresiones. La traducción italiana, en cambio, no exhibe esta pretensión de dignidad literaria y erudición. Los prejuicios que tildaban la novela de género menor e indigno para los grandes literatos eran probablemente aún más fuertes que en España. Tampoco había razón para celebrar la novela como modelo alto, o para buscarle antecedentes clásicos, contando — como contaban- con el modelo de Boccaccio. Así, en la dedicatoria se la desprestigia con el nombre de «operetta», asignándole la usual definición de regalo inapropiado para el dedicatario. El cambio de perspectiva se advierte también

traducción, las prisas a las que le sometió el editor y las disculpas por su labor: «Eccoti materia da farti un ricco vestito. Dagli la forma che più t’aggrada [...] Incontrerai qualche vocabolo sciapito; condiscilo col sale del tuo ingegno. Inciamperai in qualche periodo poco grato all'orecchie, rimediavi colla dolcezza del tuo stile; già sai che è tradozione. [...] Scusami, compatiscimi e a riservirti con migliore occasione».

7 De Rossi se mostró particularmente interesado al tema. En los preliminares de la traducción de Camus hecha por Bisaccioni llama a la obra «romanzo istorico»: una definición que para Carminati (2007: 95) se emplea por primera vez en las letras italianas.

Edad de Oro, XXXIII (2014), pp. 295-316, ISSN: 0212-0429 
en la definición de los cuatro capítulos en los que se divide el relato. Quintana los llamaba "poemas» para establecer un nuevo enlace con el noble antecedente griego, mientras que en la edición veneciana se indican simplemente con el sustantivo «libri».

Por lo demás, las palabras de De Rossi repiten una serie de estereotipos que encontramos a menudo en los preliminares de traducciones italianas de textos españoles, como el éxito del original, confirmado por sus varias ediciones: «doppo esser stato due fiate frà l'angustie de torchi della Famosa Madrid» ${ }^{8}$. Se subraya también la casualidad de la impresión, que De Rossi atribuye, entrelazándolo con el título de la obra, «a la fortuna». No falta una consideración sobre la lengua del original, aunque reducida a la frase «lo spagnuolo, che porta seco un non so che di sollevato, e non ordinario» que parece apuntar a una apreciación exótica (y no necesariamente positiva) del idioma.

Otra información interesante nos llega de la mano de la anotación del tipógrafo, quien introduce así la obra: «Eccoti, amico lettore, un romanzo spagnuolo doppo tanti francesi, che ti sei compiacciuto di leggere». Se confirma, pues, que la novela en el sentido de «romanzo», de las peripecias «bizantinas» y aventuras de amantes cortesanos, en el segundo Seicento llega a Italia sobre todo a partir de la literatura francesa (Getrevi 1986: 311; Capaldi 2001: 71-72). La traducción del español se presenta, pues, casi como una novedad.

Finalmente, el tipógrafo advierte que se dejan en español los poemas de la novela, según una costumbre frecuente en la época; basta citar, solo por poner el ejemplo más conocido, la primera edición del Chisciotte de Lorenzo Franciosini (Bernardi 1993: 153). La imposibilidad y la dificultad de la traducción del verso es un tópico al que se recurre en toda Europa. Además, eran habituales en los preliminares justificaciones como la que hallamos aquí: «Il traduttore non ha voluto cimentarsi a tradurre i versi, per non levargli i spiriti, e le vivezze». Pero hay también otra razón que lo impedía; el tipógrafo dice: «Se gl’intenderai, conoscerai quanto meriti d'essere stimato l'Auttore, se no poco ti deve importare perché questi non guastano la lettura del romanzo». Desde esta perspectiva los versos tienen, pues, un mero papel ornamental y son superfluos e irrelevantes para la comprensión y el funcionamiento global del texto, lo cual parece ser uno de los objetivos de la traducción italiana.

\section{LITERALIDAD Y SIMPLIFICACIÓN}

Las hipótesis sobre la traducción que se intuyen en los preliminares hallan su confirmación en el modus operandi de Dalla Bella. Ante todo, hay que decir que

$8 \quad$ En realidad fueron tres las ediciones madrileñas de la obra anteriores a la traducción: la princeps y las reimpresiones de 1632 y 1641. 
es difícil determinar a ciencia cierta la edición que utilizó el traductor, caso de que fuera una sola. En primer lugar, porque las variantes entre las ocho ediciones españolas que preceden a la traducción son nimias y, sobre todo, porque las lecciones de los versos españoles presentes en Il Feniso no coinciden con ninguna de las existentes. Tras analizar estas con detalle, podemos excluir que la base haya sido la princeps (se registran seis variantes diferentes). Con toda probabilidad el traductor se sirvió principalmente de la quinta reedición, impresa en 1641 (Quintana 1641): solo una variante no coincide, atribuible a un error del tipógrafo italiano (en Il Feniso falta «es» en el verso «mi amor es loco»). Además, las alusiones de De Rossi en la dedicatoria a dos ediciones madrileñas hacen pensar que la base de la traducción fue una impresa en la capital, y antes de 1654 solo hay otra, la de 1632, que descartamos de nuevo por las variantes de los poemas (no coinciden en cuatro ocasiones con las de la traducción italiana).

En general, las intervenciones del traductor son muy mesuradas, a partir de la estructura en cuatro partes, que se mantiene. En la versión inglesa, en cambio, se dividía la novela en catorce capítulos, cada uno con título propio, lo cual acentuaba la sensación de leer una antología de novelas cortas, y a la vez facilitaba la tarea de orientarse por el laberinto de las narraciones interrelacionadas (Sánchez Escribano 1987: 193-194).

Es la primera señal de la manera de traducir de Dalla Bella: la mediación, el filtro que interpone entre Quintana y el gusto del nuevo lector, no es demasiado explícito. No se trata, pues, de una recreación con interpolaciones, comentarios o glosas, como en varias traducciones de la época9 ${ }^{9}$. Dalla Bella, al contrario, traslada el texto de una manera casi siempre literal. Hay que recordar que en Italia escaseaban las verdaderas reflexiones teóricas sobre el argumento que pudiesen llevar a una uniformidad de actitudes frente al original o a resultados y prácticas compartidas. La traducción de novelas de lenguas modernas se solía considerar una actividad de escaso valor, como una especie de «artesanía de la palabra» o ejercicio de transposición. Todavía no estaba en boga el concepto de «bella infiel» y la traducción de obras en prosa, lejos de considerarse una demostración artística, se entendía como simple aproximación al original sin ambición de imitar sus primores estéticos. Por eso el traductor no podía aspirar a la gloria literaria (Greppi 1971: 57-60).

Este concepto de traducción «funcional» parece guiar a Dalla Bella. El dominico demuestra conocer bien el español, y su versión persigue ante todo la eficacia semántica. Sin embargo, a veces su adherencia textual resulta excesiva y crea calcos léxicos, semánticos y morfológicos, comunes en las traducciones de la

$9 \quad$ Piénsese tan solo en el Picariglio de Barezzi, convertido en una miscelánea tres veces más larga que el Lazarillo original (entre otros Aragone 1961: 295-299; Masala 2004: 39-60 y Pini 2012: 369-371). 
época y favorecidos por los estrechos contactos entre las dos lenguas. Esta fuerte sumisión al original indica además el escaso interés por la búsqueda formal por parte de los traductores italianos (Vuelta García 2011: 298). Así, Dalla Bella en numerosos lugares permanece más afín al significante que al significado y acoge el término italiano con la misma raíz del español, introduciendo hispanismos de uso más o menos extenso en el siglo XvII ${ }^{10}$ :

agradezco > aggradisco; conseguirían > conseguiriano; mina > mina; que a todas partes tenía > che teneva da tutte le parti; lo que pasava > ciò che passava; habiéndole respondido que sí > avendogli risposto che sì.

No obstante, la actitud de Dalla Bella no siempre se revela tan pasiva, y contrasta a veces con la mera traducción ad verbum. Su finalidad se cifra en amoldar el texto para hacerlo más apetecible y adecuado a su lector, impidiendo que tropiece en dificultades o ambigüedades expresivas. Aunque su intromisión se perciba solo en el nivel micro-textual, todos los fenómenos van en la misma dirección: la claridad y la simplificación. Sobre todo, se propone normalizar la novela y depurarla de los que, evidentemente, interpretaba como «excesos».

La primera operación en este sentido se advierte en las explicitaciones del lenguaje simbólico y culto. Muchas veces se liman las imágenes metafóricas o las perífrasis poéticas, que en la perspectiva de Quintana indican la transfiguración del mundo real en su idealización lírica. Dalla Bella suprime del todo el pasaje o, más a menudo, explica la metáfora:

dando [...] esmalte de líquido aljófar a la plata de sus canas destilado por sus ancianos ojos $(260)>0$; portátil casa de lino (247) > carozza (274); lenguas de las campanas (196) > battagli delle Campane (169); marchitó dos veces con su atrevida mano las rosas de sus mejillas (142) > con temeraria mano alla mia presenza due volte le percosse le belle guancie (50); permanece en la oculta morada del silencio (304) > può star segreto (401); hermosa afrenta del prado (118) > la più leggiadra di quei monti (2); que hizo Jacinta entre sus manos perlas (123) > che presa da Giacinta fra le mani, e cangiata in perle (9).

Se pierden también algunas referencias astronómicas y mitológicas, que confirman, una vez más, cómo Quintana se dirigía especialmente a un lector culto, familiarizado con los clásicos:

10 Se cita la versión española de la edición crítica (Quintana 2011) y se transcribe de manera diplomática el texto italiano, poniendo entre paréntesis el número de las respectivas páginas. Se dan solo pocos ejemplos de los fenómenos citados. Cuando en la traducción queda suprimida del todo una parte de texto original, se la hace seguir por $>0$. 
Volvió el alba a bordar las alfombras que Amaltea y Flora labran en las selvas (243) > Ritornata l'Alba (266);

cuando las sombras de la negra Tetis comenzaban a hacer mayores los montes y a difundir su obscuridad (263) > quando l'aria tutta era già fatta tenebrosa, ed oscura (311-312).

Otro fenómeno de simplificación de la prosa es la supresión de segmentos textuales. Son muchas las omisiones a lo largo de toda la novela, demasiadas para suponer que todas se deben a inadvertencia u olvido del traductor o del cajista. Solo en contadas ocasiones atañen a pasajes largos: se trata generalmente de pocas palabras, aisladas oraciones o breves párrafos. Sin embargo, sumándolo todo, la novela italiana es despojada de una porción bastante considerable de texto, el equivalente a unas diez páginas.

En primer lugar, Dalla Bella suprime varias de las sentencias, comentarios morales y paréntesis enciclopédicos que proliferan en el original:

razón muy propia de villanos, que, como por sí no pueden provocar a respeto, se valen de interponer la autoridad regia para ser respetados (134) > 0;

que — si bien dejando aparte que al que la lleva le ayuda Dios, sin el cual son las fuerzas más fuertes flacas y el mayor valor cobardía - aun en lo humano, si no ayuda a vencer, como la espada produce en el que va sin ella un desaliento tan grande y sobresalto tan vivo que empieza a ser principio de su vencimiento (226) > 0;

costumbre que yo alabé siempre en los antiguos franceses $(171)>0$; de quien dijo un filósofo que es ira envejecida (127) $>0$.

Es interesante observar que el traductor no interviene en las digresiones más largas de la novela, las situadas al principio de los cuatro libros: su colocación, pues, no estorba a la trama y tiene una función asimilable a la introducción de cada capítulo.

Desaparecen también varias descripciones y detalles, sobre todo cuando tienen cierto aire lírico. Así, por ejemplo, en el incipit de la novela:

Silvio, pastor por su entendimiento y por su disposición celebrado en los montes —que a la imperial Toledo vecinos son áspera población de duros robles o albergue poco culto a varias fieras - , mayoral de un mediano aprisco, dueño de un apacible rebaño — que a trechos era esmalte del prado, nieve del monte, siendo en partes aumento de las peñas (118) > Silvio Pastor tanto rinomato ne i monti all'Imperial Città di Toledo vicini;

O en otros pasajes, como:

El extremo de hermosura, el depósito de la gala, el apoyo de la bizarría, la misma agudeza en el ingenio y en amor los ojos de su padre, con gusto de mujer entendida y despejo de hombre libre (225) > una figliola di bellezza senza pari (231). 
Tanto las supresiones de partes doctrinales como las de detalles no parecen nacer de una simple voluntad de síntesis. Dalla Bella quiere evitar las interrupciones para permitir que el lector se centre en lo que más le interesa: la frenética sucesión de enredos, acciones y aventuras. Esto atestigua también una mayor preocupación de la novela barroca italiana por la unidad narrativa, advertida como auténtica exigencia de poética, y confirma, a la vez, el hecho de considerar ajeno al género novelístico el ennoblecimiento estilístico al cual sometía su página el escritor español.

Otras supresiones afectan al mundo islámico. Por supuesto, escribir en 1654 en Italia sobre el tema no era como hacerlo en España treinta años antes. Sin duda, el motivo morisco no despertaba el mismo interés ni en el plano histórico ni en el folclórico: en este sentido podemos decir que fue una manifestación casi privativa de la realidad hispánica (Mazzocchi 1994: 170-171). Así, Dalla Bella depura de su versión todos los elementos exóticos (por otra parte poco abundantes), como la mención de los cargos políticos o los trazos coloristas de la indumentaria:

visto por ellos que eran el bajá, a quien acompañaba el subaxi (277) > conosciuti da quelli (342);

arráez (275) > governatore (338);

se adornó de costoso almaizar, hermoso turbante, y todo lo demás necesario. Ciñose últimamente un limpio alfanje (251) > vestitosi dell’habito turchesco (283);

Le había hecho subaxi, que es lo mismo que alguacil mayor (274) > gl'era stata data una carica (335).

El afán de mayor inteligibilidad puede llevar también al fenómeno contrario, la amplificatio explicativa:

distantes lo que bastaba para no poder llegarse (136) > in luogo, e sito tale, che impediva loro il potersi l'uno all'altro appressare (37);

No le hallé en su casa y tierra por haberse partido a la mía (223) > Non lo ritrovai nella sua casa, e Città, per essersi partito anch'egli col medesimo fine di venir à ritrovar me (227).

Dalla Bella se sirve asimismo de otras técnicas aclaratorias; recuerda quién es el protagonista de la escena que está describiendo, explicita alusiones, perífrasis y pronombres, o se sirve de palabras más concretas y manifiestas:

doña María y las demás (302) > D. Maria, D. Leonora et Eufemia (397);

autor de su afrenta (189) > auttore del rapimento di Laura (155);

de su casa (152) > casa di D. Hippolita (73);

el amoroso dueño mío (292) > la mia diletta D. Leonora (377);

en menor edad [...], y en mayor (341) > nella fanciullezza [...] nella gioventù (486); 
lo que yo la daba (212) > per i regali che le faceva (203);

con esto (287) > con questa credenza (365);

aquello (208) > l'essersi ritrovato quivi (194);

quanto es necesario a una familia (214) > mi providdi delle suppellettili necessarie ad una famiglia (209);

desgracia (309) > vedovanza (413).

El proceso de simplificación en nombre de una narración más sobria tiene considerables repercusiones sobre el estilo. El proceso es evidente en la reordenación de la oración. En varias ocasiones Dalla Bella anticipa el sujeto, disuelve el hipérbaton o cambia la posición de los complementos en aras de una dispositio verborum más natural:

Despertó a Feniso la mala vecindad del humo (197) > la mala vicinanza del fumo destò Feniso (172);

Suspendiome el ánimo mirarla (173) > Il vederla mi sospese l'animo (118);

Siempre de la novedad es hija la admiración (185) > L'ammirazione è sempre figlia della novità (145);

Pidiendo cortésmente perdón por haberle dejado solo tanto tiempo, llegó Mahomet a Feniso (258-259) > Ritornò Maometto chiedendo cortesemente perdono a Feniso d’haverlo lasciato tanto tempo solo (301-302).

Tampoco se reproduce otro rasgo distintivo de la sintaxis de Quintana: la detractio. Muchas veces se reemplazan las partes del discurso elididas y, sobre todo, se evita el zeugma. El traductor procede así añadiendo o repitiendo verbos, preposiciones o sustantivos:

si antes cansaba con el movimiento sus miembros, ahora con imaginaciones el discurso (196) > se prima col moto stancava le membra, all'hora coi pensieri affaticava il discorso (170);

una de las cuales fue sentida de su padre y esta desgracia causa de que se tapiasen (177) $>$ in una delle quali fu sentita dal Padre e ciò fù cagione che si tennero chiuse (128);

[...] miró su triste muerte. No fue la de Félix tan breve (148) > [...] incontró una miserabile morte. Non morì Felice così presto (64).

Se reducen también otros caracteres estilísticos propios de Experiencias de amor y fortuna: verbigracia la supresión de adjetivos, recurso habitual en la prosa española del tiempo, del que Quintana abusa ${ }^{11}$ :

11 No falta, en algunas ocasiones, el fenómeno contrario, o sea la introducción de adjetivos. Sin embargo, con estas intervenciones el traductor más que una preocupación estilística parece manifestar, de nuevo, la necesidad de explicitar mejor el pasaje: en tan extraña parte (129) > in parte così disabitata e romita (23); muestras (140) > chiarissimi segni (46); le dio cuenta (183) > gli diede minutissimo conto (141). 
espaciosa sala (185) > sala (145);

una tarde del caluroso estío (247) > una sera di state (275);

pequeños troncos (257) > albero (297).

Esto lleva sobre todo a la omisión de muchos de los adjetivos apreciativos, tan frecuentes en el original y que denotaban el afán casi obsesivo de exaltar la nobleza y los valores cortesanos de los personajes y de todo lo que los rodea:

poderoso caballero (155) > Cavalliere (79);

generoso don Carlos (335) > D. Carlo (474);

ilustre Feniso (307) > Feniso (410);

noble madre (344) > Madre (495);

Madrid [...] ilustre y noble villa (244) > Madrid (269);

honrado acero (254) > spada (291).

El fenómeno es llamativo en las cadenas de adjetivos. A veces de tres se pasa a dos: apacible, clara y serena (203) > chiara, e serena (182); amarillo, flaco y macilento (220) > pallido, e macilento (221); o de dos se pasa a uno: diabólica y desatinada furia (213) > diabolico furore (207); noche tenebrosa y obscura (124) $>$ oscura notte (12). La tendencia a reducir estructuras bimembres, tan en boga en la prosa española barroca, se extiende a otras partes del discurso: sustantivos, verbos o segmentos más amplios:

furor y enojo (213) > sdegno (207);

habló y granjeó (146) > trattò (59);

a su mudable condicion, y a su fácil mudanza (196) > alla di lei mutabile natura (170); airoso despejo y atrevida resolución (332) > ardita risolutione (467);

estas bajando recias con la violencia del agua, y aquellas oponiéndose fuertes a su natural movimiento (219) $>0$.

El proceso afecta también a la correctio «A o B» popularizada por los poetas gongorinos, o las frecuentes enumerationes:

féretro, o forma de silla (123) > una seggia (10);

celda o morada (222) > cella (224);

cuyo adorno, limpieza, disposición, correspondencia y compostura (186) > i cui ornamenti (148);

sus zozobras, sus tormentos y sus penas (232) > i suoi tormenti, e le sue pene (247);

Levanteme, convalecí, esforcé la naturaleza, cobré ánimo [...] visitáronme algunos amigos (128-129) > Sorto dal letto si cominciò da alcuni amici à pratticare la mia sodisfattione (21);

quebradas razones, despedazados períodos, destroncadas palabras, tartamuda len- 
gua y anudada garganta (155) > tronche parole, con interrotti periodi, con muta lingua (79);

el cual entre el penoso dolor, el ansia indefectible y el tormento molesto (183) > il quale con incessabile affanno (140).

La reducción retórica se extiende a otras estructuras sintácticas y fonéticas que Quintana calcaba de la poesía. Con todo, no siempre el dominico italiano mantiene las paronomasias:

al parecer de pocos años, y al padecer de muchos males, aunque gallardamente dispuesto y dispuestamente gallardo (124) > Nell'apparenza faceva mostra di pochi anni, mà di molti infortuni (12);

su suspensión admirada, o su admiración suspensa (120-121) > cessò tosto in lui lo stupore (5);

No se atrevía a enviarme recados temiendo a su recato (285) > Non osava, d'inviarmi lettere, temendo la di lei modestia (361);

Allí, pues, donde la priesa acompañada del cuidado, o el sobresalto acompañado de la priesa $(197)>0$;

las repeticiones y anáforas:

Aquí de parte de Feniso eran las ansias, aquí el tormento, aquí el pesar (144) > quì si scuoprirono in Feniso angoscie, e'l dispiacere d'haver acconsentito al parere di D. Luigi (55);

aspereza [...] aspereza (148) > asprezza [...] incoltezza (66);

las aborrecía, y aborrecíalas para amarlas con más fuerza que, como el aborrecimiento [...] aborrecerte (145) > l'aborriva, et aborrivale per maggiormente amarle (che tenendo origine l'odio [...] l'odiarti (58);

o rapportationes como la correlación diseminativo-recolectiva, cuya aplicación a la prosa representaba el vértice del refinamiento pretendido por el novelista. En este caso es posible que el traductor no advirtiese la construcción sintáctica divulgada por Góngora y sus imitadores:

Hicieron a imitación de los corazones unión de los pechos, dulce aprieto de los brazos, blanda adulación de los sentidos, correspondiente silencio de las lenguas, igual alegría de los ojos, y dulce, blando, alegre y correspondiente nexo de las almas (274) $>$ Fecero ad imitatione dei cuori unione dei petti, dolce laccio delle braccia, corrispondente silenzio delle lingue, ugual allegrezza degli occhi et indissolubile nodo dell'alme (336).

Todos los cambios analizados tienen una relevante consecuencia: reducen la dimensión poética y la calidad literaria de la novela, que luce un lenguaje más frío 
y didascálico. Esta modificación (quizá salvo el último ejemplo) no parece derivar de la incapacidad de Dalla Bella para reproducir la elegancia estilística del original, ni de su dificultad para entenderlo. Sus intervenciones dejan traslucir una estrategia coherente y meditada. Dalla Bella no cree oportuno mantener un alto nivel retórico en un género como la novela: en este sentido, nada más lejos de la perspectiva italiana, en la aleación entre labor del traductor y tipografía, que los floreos de poliantea de Quintana. Hay que añadir la aversión que con frecuencia traductores y literatos italianos manifiestan hacia las formas redundantes y cargadas que censuraban en mucha de la literatura española, que culminó en la despectiva definición de «spagnolata», síntesis no solo de exageración y fanfarronería, sino también de ese estilo grandilocuente y verbosidad que había que enmendar ${ }^{12}$. Todo esto, en suma, empuja a suprimir o al menos atenuar los hábitos formales dominantes en la literatura española del primer tercio del siglo ${ }^{13}$.

$\mathrm{El}$ acercamiento al gusto y a los conocimientos del nuevo lector se advierte también en algunas intervenciones de corte cultural. De nuevo, los fenómenos de modernización o de nacionalización son prudentes: al contrario que en muchas traducciones del siglo xvII, Dalla Bella no engloba la cultura ajena en la propia con patentes sustituciones de ambientes, personajes y alusiones ${ }^{14}$.

Por lo que se refiere a la onomástica, no interviene en los nombres de ascendencia clásica, como Feniso, Teodora, Feliciana etc. Sin embargo italianiza, según las convenciones de la época, todos los demás: Félix > Felice; Luis > Luigi; Fadrique $>$ Federico; Inés $>$ Agnese; Jaime $>$ Giacopo etc. Menos evidentes son los cambios de los apellidos, que conservan un toque español: Velázquez > Velasquez; de Ávalos > d'Avalo; de Ulloa > d’Ulloa.

Lo mismo sucede con la toponimia, con la habitual adaptación de los nombres de ciudades y referencias geográficas (Zaragoza > Saragozza; Illescas > Igliesca; Col de Balaguer $>$ colle di Belaguier). El proceso de italianización extraña un poco - sobre todo al lector moderno - cuando las notas topográficas son más precisas y detalladas. Así, el barrio de «el Grao» en Valencia (Quintana 2011: 212) se convierte en «il Grado» (203) o la puerta «de Serranos» (195) en «de’ Serrani»

12 «Ho tralasciato alcuni periodi di spagnolate alquanto tediose» afirma Cattaneo, traductor del Criticón (Gambin 1995 144-146).

13 Meregalli (1974: 31) subraya que en Italia se desconocían casi por completo las obras españolas de poética literaria. Por ende solo unos pocos literatos tenían familiaridad con formas y conceptos tan populares entre los poetas y escritores del Barroco español. Por ejemplo, un texto clave como la Agudeza o Arte de ingenio de Baltasar Gracián se menciona en Italia por primera vez solo en el siglo XVIII.

14 Son ejemplares, una vez más, las traducciones de Barezzi, que traslada al ámbito italiano, modifica o suprime referencias geográficas o dichos españoles; o de Franciosini, que en su adaptación a la realidad italiana añade familias de la Italia contemporánea a las nombradas en el Quijote (Valencia 2003: 162). 
(169), mientras que el traductor decide suprimir los pasajes que se refieren a los Cigarrales de Toledo (150) o al «sagrado Pilar de Zaragoza» (344). De esta forma matiza un poco el fuerte sentido «nacional» que Quintana, según el ejemplo del Peregrino en su patria de Lope, daba a sus ambientes y personajes. Inevitablemente, el resultado es que un lector italiano no puede identificar de inmediato el espacio novelesco con lugares concretos, como sí hacía el español.

En otros «culturemas» Dalla Bella muestra una doble actitud: por un lado, se sirve de voces generales, y por el otro busca la correspondiente referencia a la realidad italiana. La finalidad es idéntica: facilitar la comprensión. Las clasificaciones métricas que preceden a los poemas de la novela son dechados de la primera técnica, con la neutralización de formas propiamente españolas. Así «espinelas», «romances», «décimas» se traducen simplemente con «versi». Se mantiene solo la palabra «sonetto» (88) por «soneto» (159), al tratarse, por razones obvias, de una forma conocida por el lector italiano (que dos veces más traduce también el español «epigrama»). Finalmente, dos traducciones de «romance» en «canzoncina» y «canzonetta» expresan la escasa consideración métrica que poseía el octosílabo desde la percepción literaria italiana, donde romance y tradición mélica vulgar debían coincidir, según nos sugieren las colecciones de poesía popular para canto.

En la vestimenta y en los cargos político-administrativos los dos usos se alternan: una generalización de las referencias: vasquiñas (175) > manti (122); caperuza (135) > capello (35); alguazil (205) > sbirro (189); corregidor (142) > Giudice (51); cabo (161) > capitano (442) discurre en paralelo con un intento de adecuación a la cultura italiana: tudesquillo (246) > pannicello (273); veguer (290) $>$ Bargello (374); privados de su casa (225) > Camerier maggiore (231); capuz (312) > capperuccio (421).

\section{CONCLUSIONES}

Tanto el paratexto como la técnica de la traducción sugieren un mismo propósito: desclasar la obra al terreno de la lectura de entretenimiento. Incluso el formato lo confirma: Il Feniso se publica en dozavo, o sea, el correspondiente en la época al libro de bolsillo, pensado para una lectura acentuadamente individual. Es modesta también la calidad gráfica, con pocos elementos decorativos y un grabado muy convencional en la portadilla. En general se reproducen, entonces, los caracteres de los volúmenes manejables y que se vendían a un precio reducido. El libro, en suma, es fiel representante de esas publicaciones "populares» y de escasa calidad tan difundidas en el siglo xviI, y que respondían a un mercado cada vez más separado y paralelo al de las ediciones de lujo (Santoro 2001: 304306). El hecho es aún más llamativo si se piensa que la primera edición del original salió en cuarto. El descuido de la manufactura de la traducción refleja, de 
nuevo, la baja concepción que se tenía en Italia de la novela «heliodoriana», que nunca formó una verdadera tradición como en España y, sobre todo, en Francia, también por falta de un autor de relieve (Courieri 2001: 507-508). Sin embargo, a la escasa consideración en los ambientes académicos correspondía un notable éxito comercial.

Esta situación - las orientaciones del mercado editorial y la colocación de la novela en el mundo literario italiano- tuvo que influir en la realización de $I l$ Feniso como producto tipográfico. La operación es evidente también en las elecciones traductivas: Dalla Bella intenta hacer asequible el texto a un público más vasto y heterogéneo, que se aleja del ideal culto que codiciaba Quintana. Sus estrategias lingüísticas y culturales apuntan entonces a ampliar el abanico de los destinatarios. En este sentido se entienden sus esfuerzos por simplificar y adecuar la novela a la cultura y a las costumbres italianas. Era este un rasgo común a muchas traducciones del género, que sacrificaban la afectación retórica sobre el altar de la claridad ${ }^{15}$. Getrevi (1986: 319-323) habla a propósito de «potencialidad democrática» de las traducciones narrativas y, citando las palabras de Franciosini y Bisaccioni, concluye que «il romanzo deve essere, almeno in potenza, comprensibile a tutti, deve poter raggiungere una più ampia fetta di lettori, deve poter soddisfare un mercato diverso».

Las fechas más tempranas de las otras traducciones europeas de Experiencias de amor y fortuna, las alusiones a los precedentes franceses en la dedicatoria y, finalmente, el examen del catálogo de los editores, nos sugieren también otras ideas. Para entender las causas de la publicación de la novela de Quintana en Italia parece necesario, pues, un enfoque internacional que considere la difusión de los textos narrativos en su dimensión europea (Getrevi 1986: 307-308; Conrieri 2011). Ampliando la perspectiva nos damos cuenta de que la traducción de Quintana corrobora una tendencia de la época. En primer lugar, confirma la duradera difusión en Italia, a nivel comercial, del llamado «romanzo eroico-galante».

El género se impuso en los años veinte y llegó a prolongarse hasta los primeros decenios de la segunda mitad del siglo. A esa altura cronológica la fuerte

15 Piénsese, por ejemplo, en las versiones italianas de Sucesos y prodigios de amor de Pérez de Montalbán y de Don Diego de noche de Salas Barbadillo, traducidas respectivamente por Biagio Cialdini (1637) y Girolamo Brusoni (1674). Por lo que se refiere a la primera obra, Cinti (1984: 585-590) ha estudiado el afán de Cialdini de regularizar la sintaxis del original: «a causa di un maggior bisogno di chiarezza, ottenuta attraverso il cambio delle costruzioni con conseguente cambio di soggetti, spezzatura del periodo con l'uso del punto fermo, la rinuncia alla dialogia, specie se accordata con zeugma [...] notiamo talvolta la distruzione di valori estetici attraverso la rinuncia di metafore e il passaggio a un eloquio piano e discorsivo». Intervenciones parecidas se hallan en la traducción de la novela de Salas Barbadillo. Vuelta García (2011: 291-298) ha analizado las supresiones de elementos que «dilatano lo sviluppo degli eventi e distolgono l'attenzione dei lettori» en una traducción que no pretende «rendere i tratti stilistici [...], limitandosi ad una letterarietà priva di eleganza». 
demanda convirtió este tipo de libros en un sistema casi automático de producción y venta, como una «moda accettata senza grandi discussioni, ormai connaturata ad una "normale" fisiologia del consumo» (Capaldi 2001: 170). Su persistente éxito obligó entonces a los editores a buscar en el exterior novelas inéditas y siempre recientes. Y las traducciones fueron no solo un medio ideal de intercambio cultural, sino también un importante soporte para el mercado italiano (Getrevi 1986: 315).

He aquí, probablemente, la razón que impulsó también la traducción de Experiencias de amor y fortuna. Una operación que Combi-La Noù creyeron rentable desde el punto de vista económico, a pesar del poco aprecio en ambientes cultos de que gozaba la novela larga española en Italia, y en general la narrativa del tiempo de los Austrias mayores. No hay que olvidar que incluso en los referentes de gran expansión europea de la prosa española — la picaresca, la novela corta o la obra de Cervantes-, no fue Italia el centro de su irradiación, sino que estos modelos tuvieron mayor fortuna en otros países (Capaldi 2001: 76-77, 174-182, 192-205). Cuando en la segunda mitad del siglo se va imponiendo un modelo extranjero en Italia, no es español: es la narrativa francesa la que condiciona el gusto y exporta un mayor número de obras (Capaldi 2001: 78, 87-89). Un influjo que, como en todos los ámbitos de la civilización, va invadiendo también la narrativa. Quintana, pues, en cuanto autor español, se antoja una excepción: por lo que se refiere a este campo, España había casi desaparecido de los mapas de Europa y aún más de los de Italia.

Podemos pensar, entonces, que Experiencias de amor y fortuna se traduce no en cuanto «obra española», sino en cuanto novela perteneciente a un determinado modelo. Tiene rasgos que podían seducir todavía al público italiano y satisfacer las exigencias del mercado: el clásico miscere utile dulci, la proliferación de tramas laberínticas y un ritmo desenfrenado de las acciones dentro de una calculada máquina literaria. Se compone de una materia convencional y consolidada (hecha de acontecimientos espectaculares como duelos, naufragios, piratas, anagnórisis, aparentes muertes, disfraces) y un fácil moralismo, repartido entre una serie de personajes estereotipados. Estos temas y estilemas que encontramos en la obra de Quintana se repetían, casi de manera formular, en muchas novelas barrocas italianas que se complacen en enumerar, más que en investigar, ambientes, acciones o el ánimo de los diversos personajes (Getto 1969). Además, Dalla Bella incrementa estos aspectos. La rapidez de la sucesión de aventuras y un dinamismo casi teatral de los hechos se enfatizan, pues, con las supresiones de pasajes descriptivos y didascálicos. Otro ingrediente que podía facilitar el acercamiento de Quintana al público italiano es su ambiente real y casi contemporáneo, que aspira, por lo menos según las convenciones novelescas de entonces, a la verosimilitud. Esto contrastaba además con el prejuicio que se tenía de las novelas españolas como 
relatos de pura invención ${ }^{16}$. La de Quintana se configura, incluso desde este punto de vista, como una acusada excepción.

Empero, a pesar de todas las cercanías entre la obra de Quintana y el romanzo italiano contemporáneo, Dalla Bella sintió la necesidad de manipular el texto para colmar las divergencias en la sensibilidad de su lector ideal y la diferente posición jerárquica que ocupaba el género en las dos culturas. Para «importar» a su país Experiencias de amor y fortuna, el traductor no tuvo más remedio que acometer una intervención llamativa: limar su brillo estético.

Todo esto, finalmente, parece poner en tela de juicio, al menos en este sector, la creencia de que al dominio político de la Corona de España en Italia correspondió también un prestigio y un influjo literario, y demuestra, por el contrario, cómo la difusión de la novela larga española, si no aislada, fue cuanto menos episódica.

Recibido: 17/09/2014

Aceptado: 25/10/2014

\section{OBRAS CITADAS}

AlbertazZI, Adolfo (1891). Romanzieri e romanzi del Cinquecento e del Seicento. Bolonia: Zanichelli.

ANTONIO, Nicolás (1996 [1738-1788]). Bibliotheca Hispana Nova sive Hispanorum scriptorum qui ab anno MD ad MDCLXXXIV floruere notitia. Madrid: Visor [Ed. facsímil Matriti: Apud Joachimum de Ibarra].

Aragone, Elisa (1961). «Barezzo Barezzi, stampatore e ispanista del Seicento». Rivista di letterature moderne e comparate, $14, \mathrm{pp} .284-312$.

Avendaño, Cristóbal de (1633). Materiale perfettissimo delle feste ordinarie, et estraordinarie della Gloriosissima Vergine Maria Madre di Dio... Composto in Lingua Spagnola dal M.R.P.M.F. Cristoforo D'Avendagno carmelitano. E trasportato nella favella italiana dal M.R.P.F. Bartolomeo Dalla Bella dominicano... In Venetia: Presso il Tomasini.

(1649). Prediche nelle solennità di Christo N.S. di D. Pietro d'Auendagno. Tradotte dallo Spagnuolo nell 'italiano dal M.R.P. fra Bartolomeo Dalla Bella. In Venetia: per Francesco Storti.

BarbiERAto, Francesco (2005). «Giovanni Giacomo Hertz. Editoria e commercio librario a Venezia nel secondo ‘600». La Bibliofilia, 107, 2, pp. 143-170.

Bernardi, Dante (1993). «Lorenzo Franciosini, primer traductor del Quijote al italiano: los problemas filológicos de la primera parte y el "caso Oudin"». Anales Cervantinos, 31, pp. 151-181.

16 Boccalini en los Ragguagli (II, 14) se refiere a las «vane fatiche de' romanzi spagnoli» para ejemplificar la diferencia con las obras de historia. Léanse también las palabras que Agricoletti en el prólogo de su Il Rodrigo pone en boca de un personaje español: «che dalla Spagna tragga origine tal nome [romanzi] non credo che vi sia dubio, e l'uso loro appresso di noi è assai frequente; ma per lo più sogliono esser favolosi, o trattati in guisa di favola» (Carminati 2007: 91-92). 
Bresadola, Andrea (2012). «En las Honras de Lope Félix de Vega y Honores extremos del doctor Juan Pérez de Montalbán: Francisco de Quintana y la oratoria fúnebre del siglo XVII». Lectura y Signo, 7, pp. 67-89.

CAPALDI, Donatella y Giovanni Ragone (2001). «La novella barocca: un percorso europeo». En Lucinda Spera (coord.), La novella barocca. Nápoles: Liguori, pp. 65-237.

CARminati, Clizia (2007). «Narrazione e storia nella riflessione dei romanzieri secenteschi». En Cinzia Carminati y Valentina Nider (coord.), Narrazione e storia tra Italia e Spagna nel Seicento. Trento: Dipartimento di Studi Letterari, Linguistici e Filologici, pp. 37-108.

CinTi, Bruna (1984). «Narrativa spagnola a Venezia (Una traduzione di Montalbán)». En Studia Historica et Philologica in honorem M. Batllori. Roma: Instituto Español de Cultura, pp. 577-593.

Cornelio, Flaminio (1749). Ecclesiae Venetae antiquis monumentis. Decadis undecimae pars prios. Venetiis: typis Jo. Baptistae Pasquali.

CONRIERI, Davide (2001). «Sulla collocazione storica della narrativa secentesca». En Lucia Strappini (ed.), I luoghi dell'immaginario barocco: atti del Convegno di Siena, 21-23 ottobre 1999. Nápoles: Liguori, pp. 501-511.

(ed.) (2011). Gli Incogniti e l'Europa. Bolonia: I libri di Emil.

Dolcetti, Giovanni (1978 [1922-1928]). Il libro d'argento delle famiglie venete: nobili, cittadine e popolari. Sala Bolognese: Forni [Ed. facsímil Venecia: Tipografia del Gazzettino-Callegari].

GAMBIN, Felice (1995). «La traduzione come servizio. In margine alla prima edizione italiana del Criticón». Quaderni di Lingue e Letterature, 20, pp. 135-150.

Getrevi, Paolo (1986). Dal picaro al gentiluomo. Scrittura e immaginario nel Seicento narrativo. Milán: Franco Angeli.

Getto, Giovanni (1969). «Il romanzo veneto nell’età barocca». En Giovanni Getto, Barocco in prosa e in poesia. Milán: Rizzoli, pp. 319-348.

Griffante Caterina, Alessia Giachery y Sabrina Minuzzi (2006). Le edizioni veneziane del Seicento: censimento. Venecia: Regione del Veneto; Milán: Bibliografica.

GREPPI, Cesare (1971). «Sulla traduzione letteraria nel Seicento italiano». Sigma, 21, pp. 52-67.

JÖCHER, Christian Gottlieb (1960 [1750-1751]). Allgemeines gelehrten Lexicon: darinne die Gelehrten aller Stände. Hildesheim: Olms [Ed. facsímil Leipzig: in Johann Friedrich Gleditschens Buchhandlung].

LePe García, María Rocío (2013a). Historia de Hipólito y Aminta de Francisco de Quintana: estudio y edición crítica [tesis doctoral]. Huelva: Universidad de Huelva. - (2013b). «La Historia de Hipólito y Aminta de Francisco de Quintana: fuentes y modelos genéricos». Etiópicas, 9, pp. 261-352.

Mancini, Albert Nicholas (1970-1971). «Il romanzo nel Seicento. Saggio di Bibliografia. Parte I-II». Studi secenteschi, 11-12, pp. 205-274 y 444-498.

Masala, Maurizio (2004). Il Picariglio castigliano di Barezzo Barezzi: una versione seicentesca del Lazarillo de Tormes. Roma: Bulzoni.

MazzocchI, Giuseppe (1994). «La novela morisca y su relación con la novela barroca italiana: Anton Giulio Brignole Sale y la 'Storia spagnuola'». Revista del Departamento de Filología Moderna (Ciudad Real), 5, pp. 163-181. 
MAzzocchi, Giuseppe (2005). «Il paratesto nelle traduzioni letterarie di testi spagnoli (secoli XVI-XVII)». En Marco Santoro y Maria Gioia Tavoni (coord.): I dintorni del testo. Approcci alle periferie del libro. Roma: Edizioni dell’Ateneo, pp. 393-412.

Mazzucchelli, Giammaria (1760). Gli scrittori d'Italia. Cioè Notizie storiche, e critiche intorno alle vite, e agli scritti dei letterati italiani. Brescia: Giambatista Bossini.

Meregalli, Franco (1974). Presenza della letteratura spagnola in Italia. Florencia: Sansoni.

Michel, Suzanne Paul y Paul-Henri Michel (1967-1984), Répertoire des ouvrages imprimés en langue italienne au XVIIe siècle conservés dans les bibliothèques de France. París: Centre National de la Recherche Scientifique.

Mirto, Alfonso (1989). «Librai veneziani nel Seicento: i Combi-La Noù ed il commercio con l'estero». La Bibliofilia, 91, 3, pp. 287-305.

Niseno, Diego (1634). La sete che pati Christo nostro redentore nella croce. Il tradimento di Giuda. Assonti predicabili del P.F. Diego Nisseno abbate del monastero del gran Basilio di Madrid. Tradotti dal M.R.P.F. Bartolomeo Dalla Bella domenicano del convento di SS. Gio. e Paolo di Venetia... In Venetia: presso il Sarzina.

PINI, Donatella (2012). «Barezzi autore, traduttore, editore di romanzo spagnolo e dintorni». En Valentina Nider (coord.), Il prisma di Proteo. Riscritture, ricodificazioni, traduzioni fra Italia e Spagna (sec. XVI-XVIII). Trento: Dipartimento di Scienze Filologiche e Storiche, pp. 353-371.

Quintana, Francisco de [Francisco de las Cuevas] (1626). Experiencias de amor y fortuna. En Madrid: por la Viuda de Alonso Martín.

- (1636) [Francisco de las Coüeras]. Le Fenise: histoire espagnole; ou se voyent les diuers effets de l'amour et de la fortune. A Paris: chez Antoine de Sommaville. (1641). Experiencias de amor y fortuna. En Madrid: en la Imprenta del Reyno. (1651) [Francisco de la Coveras]. The History of Don Fenise. A new Romance. Written in Spanish by Francisco de la Coveras, and Now Englished by a Person of Honor. Londres: Printed for Humphrey Moseley.

(1654). Il Feniso, overo Avvenimenti d'Amore e di Fortuna, portato dalla lingua spagnuola da Bartolomeo Dalla Bella. In Venetia: Per Combi e La Noù. (2011). Experiencias de amor y fortuna, Andrea Bresadola (ed.). Sevilla: Unia.

SÁnchez Escribano, Francisco Javier (1987). «La versión inglesa de Experiencias de Amor $y$ de Fortuna de Francisco de Quintana (1651)». En Julio César Santoyo e Isabel Verdaguer (coord.), De clásicos y traducciones: versiones inglesas de clásicos españoles (ss. XVI-XVII). Barcelona: Promociones y Publicaciones Universitarias, pp. 189-204.

SANTORo, Marco (2001). «Caratteristiche e valenze dell'editoria barocca». En Lucia Stra-ppini (coord.), I luoghi dell'immaginario barocco: atti del Convegno di Siena, 21-23 ottobre 1999. Nápoles: Liguori, pp. 295-306.

SANTORO, Marco y Silvia Zannini (2007). «La fortuna editoriale delle opere spagnole in Italia nei secoli XVI e XVII: primi risultati di una campionatura ed appunti paratestuali». En María de las Nieves Muñiz Muñiz, La traduzione della letteratura italiana in Spagna (1300-1939): traduzione e tradizione del testo, dalla filologia all'informatica. Atti del Primo Convegno Internazionale (13-16 aprile 2005). Barcelona / Florencia: Universitat de Barcelona - Franco Cesati, pp. 443-466. 
Ulvioni, Paolo (1977). «Stampatori e librai a Venezia nel Seicento». Archivio Veneto, 108, pp. 93-124.

VALEncia, María Dolores y Victoriano Peña (2003). «Teoría y práctica de la traducción hispano-italiana en el siglo xvII: reflexión gramatical y labor traductora de Lorenzo Franciosini». En José Antonio Sabio Pinilla y María Dolores Valencia (coord.), Seis estudios sobre la traducción en los siglos XV y XVII. Granada: Comares, pp. 119-167.

Vuelta García, Salomé (2011). «La narrativa spagnola e l’accademia degli Incogniti: le traduzioni di Girolamo Brusoni». En Davide Conrieri (coord.), Gli Incogniti e l'Europa. Bolonia: I libri di Emil, pp. 277-313.

Zimič, Stanislav (1975). «Francisco de Quintana, un novelista olvidado, amigo de Lope de Vega». Boletín de la Biblioteca Menéndez Pelayo, 51, 1-4, pp. 169-232. 


\section{. \\ LA NOVELA ESPAÑola EN LA ITALIA DEL SIGLO XVII: \\ el caso de Il Feniso de Francisco de Quintana}

Resumen: Se estudia la versión italiana —realizada por Bartolomeo Dalla Bella (1654) _ de la novela Experiencias de amor y fortuna (1626) de Francisco de Quintana, tanto desde un punto de vista traductivo como desde una perspectiva más amplia, colocándola en el panorama de la narrativa española barroca en Italia. El análisis del paratexto y de la estrategia de traducción permite individuar un propósito común: matizar la erudición, el afán moralizador y el refinamiento estilístico del original. Asistimos así a una constante simplificación de todos los preciosismos léxicos, sintácticos y culturales. El resultado es que la novela se desclasa a simple lectura de entretenimiento para incrementar el abanico de lectores. Esto demuestra, por un lado, la distinta posición jerárquica que ocupaba el género en las dos culturas y confirma, por otro, el éxito en Italia de este tipo de narrativa de aventuras.

Palabras claves: Francisco de Quintana, Il Feniso, Bartolomeo dalla Bella, Novela cortesana-bizantina, Traducción áurea, Paratexto.

\section{THE SPANISH NOVEL IN XVII-CENTURY ITALY:} the CASE of Il Feniso by Francisco de Quintana

AвSTRACT: The article studies the Italian version —edited by Bartolomeo Dalla Bella (1654) — of the novel Experiencias de amor y fortuna (1626) by Francisco de Quintana, both from a translation point of view, and looking at it from a broader perspective, the one of its placement into Baroque Spanish narrative landscape in Italy. The analysis of the paratext and the translation strategy enables to identify a common objective: to fade the erudition, moralistic significance and stylistic refinement of the original. What we see is a constant simplification of each lexical, syntactical and cultural preciosity. The result is a downgrading of the novel to a simpler reading aimed to entertain, in order to broaden the reading audience. This shows, on one side, the different hierarchical position that the genre occupied in the two cultures, and on the other confirms the success of such adventure narrative in Italy.

Keywords: Francisco de Quintana, Il Feniso, Bartolomeo dalla Bella, Byzanthine-courtly novel, Golden Age translation, Paratext. 
Evangelina Rodríguez Cuadros (Universitat de València)

Novela cortesana, novela barroca, novela corta: de la incertidumbre al canon .9

Mita Valvassori (Universidad de Los Lagos)

El modelo narrativo del Decamerón en la Edad de Oro: una vieja historia .21

Antonio Gargano (Università degli Studi di Napoli Federico II)

«Difficile est proprie communia dicere»: el género de la novella entre

Boccaccio y Cervantes

Guillermo Carrascón (Università degli Studi di Torino)

Apuntes para un estudio de la presencia de Bandello en la

novela corta del siglo XVII

Leonardo Coppola (Università degli Studi «G. d'Annunzio» di Chieti-Pescara)

La proyección de Straparola en la novela española del Siglo de Oro desde una perspectiva editorial

Mireia Aldomì García

Didactismo, género literario y lector en Giraldi Cinzio.

María Jesús Zamora (Universidad Autónoma de Madrid)

«...En tiempo menos discreto que el de agora, aunque de hombres más sabios, se

Ilamaban a las novelas cuentos». La novela corta y el cuento en el Siglo de Oro.....109

Marcial Rubio (Università degli Studi «G. d'Annunzio» di Chieti-Pescara)

La contribución de Cervantes a la novela barroca: la ejemplaridad. .125

PIERRe Darnis (Université Bordeaux Montaigne)

La fuerza de la sangre, La ilustre fregona $y$ Las dos doncellas: ¿tres tipos

folclóricos?

María Soledad ArRedondo (Universidad Complutense de Madrid)

De La gitanilla $a$ La sabia Flora malsabidilla. El género, el personaje

y el matrimonio

Antonella Gallo (Università degli Studi di Verona)

Fabulaciones en equívocos burlescos: la Chrónica del monstro imaginado (1615)

de Alonso de Ledesma y novela corta barroca

David GonZález Ramírez (Universidad de Málaga)

El filósofo del aldea (1625) de Baltasar Mateo Velázquez: recepción textual

e hipótesis autorial.

Jonathan BRAdBury (University of Exeter)

La narrativa breve en la miscelánea del siglo XVII 
Cristina Castillo Martínez (Universidad de Jaén)

«La fuente del desengaño»: de las Noches de invierno de Eslava a la Tercera

Diana de Tejeda.

María Zerari (Université Paris-Sorbonne, CLEA)

Furor in fabula: La cruel aragonesa de Castillo Solórzano (o de la dama monstruo).. 241

Giulia Giorgi (Università degli Studi di Ferrara)

Alonso de Castillo Solórzano reescritor de sí mismo: algunas notas sobre los

Escarmientos de amor moralizados y el Lisardo enamorado .257

Angela Fabris (Alpen-Adria-Universität Klagenfurt)

El diálogo con el público y los espacios reales y de maravilla en

Casos prodigiosos y cueva encantada de Juan de Piña .267

María Rocío LePe García (IES San Sebastián, Huelva)

La traducción inglesa de Hipólito y Aminta: una adaptación

con fines comerciales 281

Andrea Bresadola (Università degli Studi di Udine)

La novela española en la Italia del siglo XVII: el caso de Il Feniso

de Francisco de Quintana

José Teruel (Universidad Autónoma de Madrid)

El triunfo del Desengaño. Marco y desengaño postrero de la Parte segunda

del Sarao y entretenimiento honesto, de María de Zayas

Nieves Romero-Díaz (Mount Holyoke College)

Lecturas alternativas en la Novela del fin bueno en mal principio

de doña Ana Francisca Abarca de Bolea.

Shifra Armon (University of Florida)

Compromiso y distanciamiento en La Venus de Ferrara

de Mariana de Carvajal Saavedra

Mechthild Albert (Rheinische Friedrich-Wilhelms-Universität Bonn)

Las "noches": un subgénero novelístico en perspectiva comparada.... .365

Fernando Copello Jouanchin (Université du Maine, Le Mans)

El mueble en la novela corta del Siglo de Oro: algunas reflexiones

en torno a la cama

Ilaria Resta (Università del Salento):

De la novella al entremés pasando por la novela corta: reescrituras del cuento

La gara delle tre mogli del Cieco di Ferrara. 


\section{EDAD DE ORO}

Revista de Filología Hispánica XXXIII

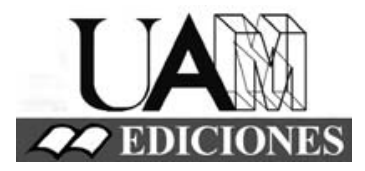




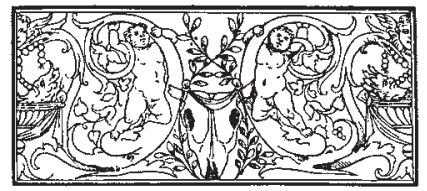

\section{Edad de Oro. Revista de Filología Hispánica}

ISSN: 0212-0429

Dirección:

Teodosio Fernández

Secretaría y edición:

José Ramón Trujillo

Coordinador del volumen XXXIII:

Rafael Bonilla Cerezo

Comité científico internacional:

Carlos Alvar (Univ. de Ginebra)

Ignacio Arellano (Univ. de Navarra)

Javier Blasco (Univ. de Valladolid)

Alberto Blecua (UAB)

Jean Canavaggio (Univ. de París X)

Laura Dolfi (Univ. de Turín)

Aurora Egido (Univ. de Zaragoza)

Víctor García de la Concha (RAE)

Luciano García Lorenzo (CSIC)

Joaquín González Cuenca (Univ. de Castilla-

La Mancha)

Agustín de La Granja (Univ. de Granada)

Begoña López Bueno (Univ. de Sevilla)

Michel Moner (Univ. de Toulouse III)

Joan Oleza (Univ. de Valencia)

Alfonso Rey (Univ. de Santiago)

Lina Rodríguez Cacho (Univ. de Salamanca)

Leonardo Romero Tobar (Univ. de Zaragoza)

Aldo Ruffinatto (Univ. de Turín)

Lía Schwartz (City University of New York)
Redacción y admisión de originales:

Teodosio Fernández

Edad de Oro

Departamento de Filología Española

Universidad Autónoma de Madrid

28049 Madrid (España)

Tfno.: +0034 914974090

correo: teodosio.fernandez@uam.es

Distribución, suscripción y venta:

Servicio de Publicaciones de la UAM

Universidad Autónoma de Madrid

28049 Madrid (España)

Intercambio de publicaciones:

Biblioteca de la Facultad de Filosofía y

Letras (UAM)

Universidad Autónoma de Madrid

28049 Madrid (España)

Han colaborado en este volumen:

Departamento de Filología Española (UAM)

Facultad de Filosofía y Letras (UAM)

Proyecto I+D FFI2013-41264-P La novela

corta del siglo XVII: estudio y edición (y II)

Edad de Oro se recoge en las siguientes bases de datos: SCOPUS, MLA Database, HLAS, Latindex, PIO-Periodical Content Index, ISOC, Dialnet, MIAR, ERIH, DICE, Sumaris CBUC, Ulrich's. Se encuentra evaluada en CIRC: A; INRECH: primer cuartil, posición 6 de 50; MIAR difusión ICDS live: 9.977; SCImago Journal \& Country Rank: H Index 2, SJR 0,101, Q4; RESH índice de impacto: 0.162; ERIH: A INT1; Carhus Plus+: B. 